\title{
Entanglement Molecular Weight
}

\author{
Yuichi Masubuchi ${ }^{* * *, \dagger}$, Yuya Doi ${ }^{* *}$, and Takashi UneYAma, ${ }^{* * *}$ \\ "Center of Computational Science \\ ${ }^{* *}$ Department of Materials Physics, Nagoya University, Nagoya 464-8603, Japan
}

(Received : June 9, 2020)

\begin{abstract}
In some literature, entanglement molecular weight $M_{\mathrm{e}}$ is discussed as a material property that characterizes magnitudes of entanglement for polymeric liquids. However, $M_{\mathrm{e}}$ was introduced as a model parameter for theories that explain the plateau modulus, and the value is model dependent. A value of $M_{\mathrm{e}}$ may induce misleading analysis if it is presented without noting the underlying theoretical model. Meanwhile, attempts in molecular simulations allow us to extract $M_{\mathrm{e}}$ from a snapshot of entwining molecules via the topological analysis. However, the relation has not been fully clarified yet between the extracted network and the entangled polymer dynamics; the latter is the retarded molecular motion observed for dense polymeric liquids with high molecular weight. Analysis of the reduction of entanglement density under deformations is also a matter of discussion since some molecular theories can reproduce non-linear rheology with stable entanglement density.
\end{abstract}

Key Words: Viscoelasticity / Polymers / Rheology / Simulation / Plateau modulus

\section{ENTANGLEMENT OF POLYMERS}

Entanglement phenomenon represents the retarded molecular motion observed in dense polymeric liquids with high molecular weight ${ }^{1)}$. For polymer melts with low molecular weight, the diffusion coefficient $D$ and the viscoelastic relaxation time $\tau$ depend on the molecular weight $M$ described as $D \sim M^{-1}$ and $\tau \sim M^{2}$. These behaviours can be explained by the Rouse theory ${ }^{2)}$, in which the effects of surrounding polymers are embedded into the friction constant of the bead-spring chain. As the molecular weight increases, the power-law exponent changes as $D \sim M^{-2.2}$ and $\tau \sim M^{3.5}$, demonstrating a significant slow-down of the molecular motion ${ }^{1,3-5)}$. Polymeric systems in such a state are so-called "entangled polymers", and the correlation between the polymers that induces the retarded molecular motion is referred to as entanglement coupling ${ }^{1)}$.

The fundamental mechanism of the retardation has not been fully clarified yet. There have been reported no structural transition at the critical molecular weight for entanglement ${ }^{1,3-5)}$. Meanwhile, it has been established that uncrossability between chains has been specified to play a significant role. The coarse-grained molecular simulations, in which the uncrossability is guaranteed via the excluded volume,

\footnotetext{
$\dagger$ Corresponding author.

E-mail : mas@mp.pse.nagoya-u.ac.jp

Tel: +81-52-789-2551
}

reproduce the entangled polymer dynamics ${ }^{6,7)}$. Attempts have been made from the topology between chains or the magnitude of entwining among the chains ${ }^{8-12)}$.

Meanwhile, some theoretical models have attained remarkable success with hypothetical setup for the polymer motion ${ }^{4,5,13-15)}$. In these models, the polymers move under dynamic constraint, for which a particular characteristic length is assumed. In the temporal network model ${ }^{16)}$ and the sliplink model ${ }^{17)}$, the polymer segments are trapped at specific nodes distributed in space with a certain correlation length. In the tube model ${ }^{18,19)}$, the polymer dynamics is represented by a single chain motion confined in a tube that has a specific diameter. The rubber-like plateau in the viscoelastic relaxation hints such a characteristic length since the modulus can be interpreted as thermal energy per characteristic volume.

The term "entanglement molecular weight", usually referred to as $M_{\mathrm{e}}$, is a chemistry dependent parameter for the theoretical models mentioned above. In what follows, we note that the value of $M_{\mathrm{e}}$ depends on the underlying theoretical model, even if the plateau modulus is experimentally specified $^{20)}$. Besides, we review some attempts in molecular simulations, in which the entanglement network is extracted from intertwining polymers.

\section{Me FROM GN}

As mentioned above, $M_{\mathrm{e}}$ is experimentally obtained 
from the plateau modulus $G_{\mathrm{N}}$. The most widely used definition is as follows.

$$
M_{\mathrm{e}}=\frac{\rho R T}{G_{\mathrm{N}}}
$$

Here, $\rho$ is the polymer density, $R$ the gas constant, and $T$ the temperature. We wish to note that a model-dependent constant $A$ is hidden in equation 1 as

$$
M_{\mathrm{e}}=A \frac{\rho R T}{G_{\mathrm{N}}}
$$

The expression given in eq. 1 stands for the case of $A=1$. We shall review the value of $A$ that depends on the molecular model behind. Note that we follow the binary assumption of entanglement to have four diverging strands from one network node, and the effect of functionality is not discussed below.

The relation between modulus and molecular weight of the network strand has been well-investigated for rubber elasticity $^{21,22)}$. Indeed, the notation of eq. 1 was introduced according to the analogy to the affine network theory ${ }^{23)}$, in which the strand molecular weight $M_{\mathrm{C}}$ is used instead of $M_{\mathrm{e}}$. This theory assumes that the network deforms affinely, and the node position does not fluctuate at all, as schematically described in Fig. 1(a). For entangled polymers, Ferry ${ }^{1)}$ utilized $A=1$ in his book to tabulate the value of $M_{\mathrm{e}}$ determined from the data for $G_{\mathrm{N}}$.

Although the affine assumption is convenient, the idea is somewhat incompatible to the use of entropic spring for each network strand. Namely, the entropic spring comes from thermal fluctuations of the Kuhn segments. If such fluctuations are allowed, the position of network nodes must fluctuate at least in the same order of magnitudes with the segments, as depicted in Fig. 1(b). The phantom network theory ${ }^{24,25)}$ takes account of such fluctuations, and the theory gives $A=1 / 2$. Although this value of $A$ has not frequently been employed for entangled polymers, it is consistent with the value of $M_{\mathrm{e}}$ determined by the primitive path analysis, as mentioned later.

For the entanglement network, the fluctuation along the chain contour comes into play. In the tube model proposed by Doi and Edwards ${ }^{3)}$, the tube deforms affinely, whereas the segments are allowed to slip inside the tube, as shown in Fig. 1 (c). This slippage gives $A=4 / 5$, and Fetters ${ }^{26)}$ tabulated the value of $M_{\mathrm{e}}$ according to this $A$ value.

The realistic situation would be with both of the fluctuations explained above. Namely, fluctuations are considered for the position of the network nodes and the number of segments on each network strand, as shown in Fig. 1 (d). For the slip-tube model proposed by Rubinstein and Panyukov ${ }^{22)}$, the value of $A$ for such a case is $A=4 / 7$. This value is close to the empirical parameter for the multi-chain slip-link model determined as $A=2 / 3$ by Masubuchi et al. ${ }^{27,28)}$.

There is another class of molecular models, so-called slip-spring models ${ }^{29-32)}$. In this framework, the entanglement is replaced by a spring that connects two chains, as seen in Fig. 1 (e). This modelling of entanglement induces additional

(a)

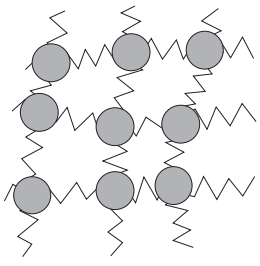

(b)

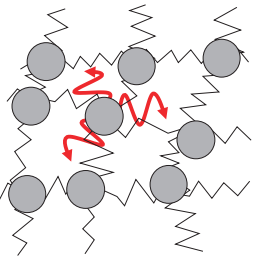

(c)

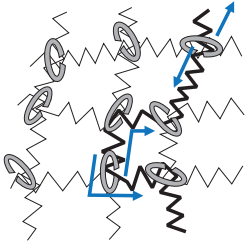

(d)

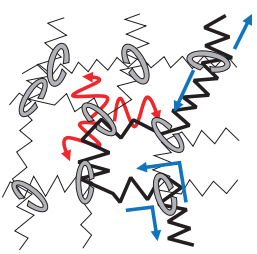

(e)

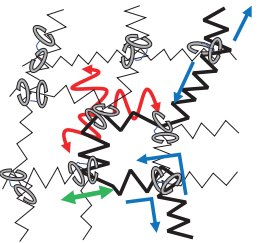

Fig. 1 Schematic representation of theoretical models for the elasticity of a polymer network. Fig (a) depicts the affine network model, in which the network deforms affinely without fluctuations. In the phantom network model shown in Fig (b), the network nodes are allowed to fluctuate around its equilibrium position, as indicated by red curved arrows. The tube model can be replaced by the slip-link network seen in Fig (c), where the network nodes do not fluctuate in space, whereas the chain slides along its contour through the network nodes, as shown by blue arrows. Fig (d) represents the multi-chain slip-link model that takes account of the fluctuations for the node position and the chain sliding. The multi-chain slip-spring model adds the other fluctuations to the slip-link model by the virtual springs that connect two chains, as shown in Fig (e). 
Table I $A$ values in eq. 2 for various network models.

\begin{tabular}{llc}
\hline \multicolumn{1}{c}{ Model } & \multicolumn{1}{c}{ Fluctuations } & $A$ value \\
\hline Affine network & & 1 \\
Phantom network $^{24,25)}$ & N/A & $1 / 2$ \\
$\begin{array}{l}\text { Tube model } \\
\text { (Slip-link network) }\end{array}$ & Brownian motion of nodes & $4 / 5$ \\
$\begin{array}{l}\text { Slip-tube model } \\
\text { (Multi-chain slip-link network) }\end{array}$ & Chain sliding & $4 / 7$ \\
Multi-chain slip-spring model $^{34)}$ & Brownian motion of nodes, chain sliding, and node flexibility & $2 / 3 *$ \\
\hline & *Empirically obtained & \\
\hline
\end{tabular}

Table II $M_{\mathrm{e}}$ values of polymers used for some models.

\begin{tabular}{lcccc}
\hline \multicolumn{1}{c}{ Model } & PS & PI & PB & Bead-spring model* \\
\hline Affine network $^{1)}$ & 18100 & - & 2500 & - \\
Tube $^{36-38)}$ & 14470 & 4820 & 1930 & 72 \\
Multi-chain slip-link $^{28,34)}$ & 11000 & 3500 & 1600 & 40 \\
Multi-chain slip-spring $^{34,35,39)}$ & 4400 & 1400 & - & 28 \\
\hline
\end{tabular}

*For the standard model proposed by Kremer and $\mathrm{Grest}^{40)}$.

fluctuations to the abovementioned models, in which the bundled chains share the position of entanglement. Uneyama ${ }^{33)}$ has recently derived $A \sim 0.24$, which is close to the values empirically employed for the multi-chain slip-spring simulations.

The $A$ values mentioned above are summarized in Table I. Because $G_{\mathrm{N}}$ and $\rho$ are defined experimentally, the value of $A$ affects the estimation of $M_{\mathrm{e}}$. Table II shows the $M_{\mathrm{e}}$ values. Apart from the data taken from Ferry's book ${ }^{1)}$, the values are used for the fitting of theoretical results of $G^{\prime} G^{\prime \prime}$ data to experiments. Although not in a fully quantitative agreement, the $M_{\mathrm{e}}$ values are consistent with $A$ values in Table I. For instance, if we compare the value of $M_{\mathrm{e}}$ for polystyrene estimated from the tube theory $(A=4 / 5)$ and the slip-spring model $(A \sim 0.24)$, the value for the latter is $30 \%$ of the former.

One may argue that the small $M_{\mathrm{e}}$ values in some models are inconsistent with the transition between unentangled and entangled behaviours seen for the molecular weight dependence of the diffusion and the relaxation time. Ferry ${ }^{1)}$ summarized that the critical molecular weight for the transition, $M_{c}$, is $M_{c} \sim 2 M_{\mathrm{e}}$. However, this relation was obtained for $A=1$, and it depends on the $A$ value. In the other extreme, for the slip-spring simulations, $M_{c} \sim 8 M_{\mathrm{e}}$ is observed ${ }^{34,35)}$. This result can be rationalized by the fact that the fluctuations weaken the constraint of entanglement to the chain dynamics.

We note that the value of $A$ is independent of the polymer density. For each model, with the number density of the network strand $v$, the unit of modulus can be defined as $G_{0}=v k_{\mathrm{B}} T$, in a sense that the modulus is the density of thermal energy. According to this expression, one may argue that the value of $A$ is a matter of conversion for the unit of length and the node number in the unit volume. However, we emphasize that $A$ is not related to the conversion of density. Indeed, $G_{0}$ does not corresponds to the plateau modulus $G_{\mathrm{N}}$, and the parameter $A$ compensates the difference as $G_{\mathrm{N}}=A G_{0}$. For instance, for the multi-chain models, the conversion of density is excluded ${ }^{34)}$ in the values of $A$ shown in Table II.

In the discussion above, the plateau modulus $G_{\mathrm{N}}$ is regarded as a well-defined experimental quantity. However, the values of $G_{\mathrm{N}}$ reported in the literature are in a spread. For instance, Liu et $a .^{41)}$ mentioned that the literature value of $G_{\mathrm{N}}$ for polyethylene ranges from $1.1 \mathrm{MPa}$ to $2.6 \mathrm{MPa}$. The reason for this scattering is i) the difference of the estimation method, ii) the molecular weight dependence of $G_{\mathrm{N}}$, and iii) the effect of the molecular weight distribution.

The most reliable and widely used method is the socalled integration method, in which $G_{\mathrm{N}}$ is obtained as the integration of $G^{\prime \prime}$ over the terminal relaxation peak ${ }^{1)}$. The advantage of this method is that the obtained $G_{\mathrm{N}}$ is insensitive to the molecular weight distribution if the terminal relaxation is sufficiently separated from the Rouse modes. There are a few other methods that are practically convenient. Namely, $G_{\mathrm{N}}$ is determined from $G^{\prime}$ at the frequency where $G^{\prime \prime}$ shows the minimum ${ }^{1)}$, the maximum ${ }^{42)}$, or the cross-over ${ }^{11)}$ with $G^{\prime}$. For scarcely entangled systems, some of these methods are inapplicable because $G^{\prime \prime}$ does not exhibit clear bottom nor peak. Besides, $G_{\mathrm{N}}$ from these methods is strongly affected by the molecular weight distribution. Meanwhile, for 
well-entangled and monodisperse systems, the $G_{\mathrm{N}}$ values obtained from the various methods agree with each other within ca. $10 \%$ error $^{41)}$.

The tabulated values of $G_{\mathrm{N}}$ in the literature can be regarded as the value at the long-chain limit. Meanwhile, $G_{\mathrm{N}}$ depends on the molecular weight because of the structural and kinetic reasons. Flory ${ }^{43}$ has argued that a fraction of cross-linkers is formed inter-molecularly, and it does not contribute to the modulus. Based on this idea, the following equation for the affine network theory can be derived.

$$
G_{\mathrm{N}}=G_{\mathrm{N}}^{\infty}\left(1-\frac{2 M_{\mathrm{e}}^{\mathrm{AN}}}{M}\right)
$$

Here, $G_{\mathrm{N}}^{\infty}$ is the value of $G_{\mathrm{N}}$ at the long chain limit, and $M_{\mathrm{e}}^{\mathrm{AN}}$ is the value of $M_{\mathrm{e}}$ based on the affine network theory $(A=1)$. Meanwhile, for the tube model, Doi ${ }^{44)}$ proposed the following relation based on the contour-length fluctuation theory.

$$
G_{\mathrm{N}}=G_{\mathrm{N}}^{\infty}\left[1-C\left(\frac{M_{\mathrm{e}}^{\mathrm{T}}}{M}\right)^{1 / 2}\right]
$$

Here, $M_{\mathrm{e}}^{\mathrm{T}}$ is $M_{\mathrm{e}}$ from the tube theory $(A=4 / 5)$, and $C$ is a constant. Masubuchi et al $^{27,28)}$ have proposed another equation for the multi-chain slip-link model considering the cycle rank of the network.

$$
G_{\mathrm{N}}=G_{\mathrm{N}}^{\infty}\left(1-\frac{3 M_{\mathrm{e}}^{\mathrm{MS}}}{M}\right)
$$

Here, $M_{\mathrm{e}}^{\mathrm{MS}}$ is $M_{\mathrm{e}}$ for the multi-chain slip-link model $(A=2 / 3)$. Nevertheless, the molecular weight dependence of $G_{\mathrm{N}}$ has not been established experimentally. Figure 2 shows an example of the comparison between experimental data and the theory by Masubuchi et $a l .^{27,28)}$, demonstrating the scattering of the

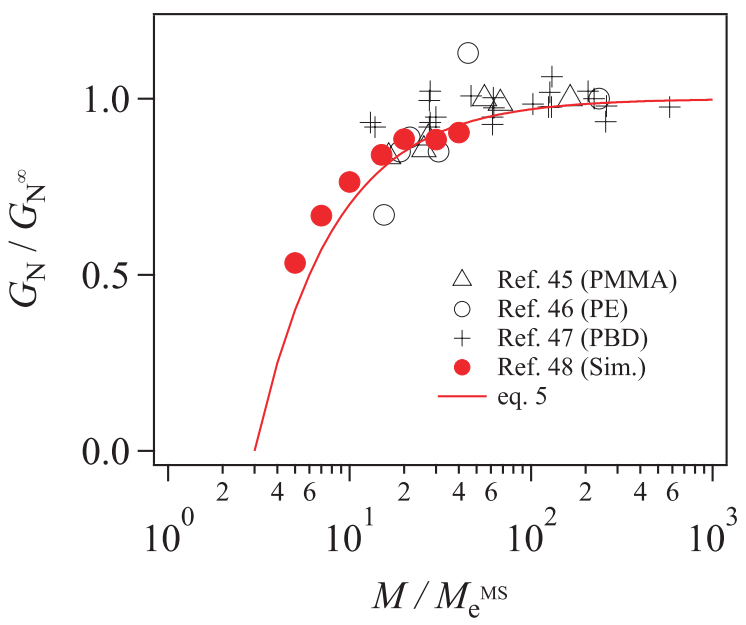

Fig. 2 Molecular weight dependence of $G_{\mathrm{N}}$. Red curve shows the theoretical prediction of eq. 5. Black symbols are experimental data from literature ${ }^{45-47)}$. Red circle is the result from the multi-chain slip-link simulation $^{48)}$. experimental values ${ }^{45-47)}$ in low $M$ regime. Besides, this plot implies that the convergence of $G_{\mathrm{N}}$ to $G_{\mathrm{N}}^{\infty}$ is seen in highly entangled systems, in which $M \gtrsim 100 M_{\mathrm{e}}^{\mathrm{MS}} \sim 50 M_{\mathrm{e}}^{\mathrm{AN}}$. This condition for $M$ is similar to that mentioned by Liu et al. for the evaluation of $G_{\mathrm{N}}$ by the integration method. However, their condition comes from a different aspect, in which the Rouse and the terminal relaxations must be well-separated.

\section{Me FROM MOLECULAR SIMULATIONS}

Although the value of $M_{\mathrm{e}}$ is hardly obtained experimentally, it could be measured in molecular simulations. The widely-used method is relying on the mean-square-displacement, in which a few inflection points are predicted before exhibiting the normal diffusion according to the tube theo$\mathrm{ry}^{3,40)}$. One of such inflections gives the squared tube diameter that corresponds to the chain dimension of a segment with the molecular weight of $M_{\mathrm{e}}$. However, the value thus obtained is smaller than that estimated from the modulus (with $A=4 / 5)^{49)}$.

There are a few attempts, in which the entanglement network is extracted among the entwining chains in the simulation box. The pioneering work is the so-called primitive path analysis (PPA) proposed by Everaers et al. ${ }^{50)}$. For a well-equilibrated snapshot of the molecular simulation, they fixed all the chain ends in space, and tightened the chains up by reducing the temperature and removing the intrachain excluded volume. Then they obtained the network of taut chains tangled with the other chains. Assuming that the fastened chains thus obtained corresponds to the primitive chain considered in the tube model, they calculated the chain statistics to estimate the average molecular weight of the tube segment. They reported that the obtained value is consistent with $M_{\mathrm{e}}$ for $A=4 / 5$. They have elaborately reported that the PPAbased $M_{\mathrm{e}}$ follows the packing length theory for various polymers ${ }^{51)}$. Although this method prepares the statistics of taut chains as a whole, the network topology is hardly analyzed. In other words, one cannot specify individual entanglement points. For this issue, the other methods have been proposed to extract entanglements from the minimization of contour length ${ }^{52-54)}$. Interestingly, the value of $M_{\mathrm{e}}$ obtained from this method is consistent with $A=1 / 2$. Everaers ${ }^{55)}$ explained this difference by the thermal fluctuations around the entanglement, as an analogy to the rubber theories mentioned in the previous section. Namely, the $M_{\mathrm{e}}$ obtained from PPA is the value for the energetically minimized network without fluctuations. In contrast, the value obtained from the topological analysis is for a snapshot of the fluctuating network. This idea 
could be rationalized by the results from the iso-configurational analysis ${ }^{56)}$, where $M_{\mathrm{e}}$ is defined from the magnitudes of fluctuations, and the obtained value is consistent with $A=1 / 2$.

An intriguing outcome from the topological analysis is the distribution of $M_{\mathrm{e}}$. Based on the classical theories ${ }^{57,58)}$, in which the binary entanglement occurs along the chain randomly, the single-exponential function has been proposed. However, the distribution obtained from the topological anal$\mathrm{ysis}^{53)}$ has a peak, as shown in Fig. 3. A similar distribution has been reported from the multi-chain slip-link simulation ${ }^{59}$. Tzoumanekas and Theodorou ${ }^{53)}$ proposed the following functional form for this distribution by considering blocking events, which prevents clustering of entanglements along the chain.

$$
P(n)=\frac{1}{\langle n\rangle} \frac{1}{\alpha-\beta}\left[\exp \left(-\frac{n /\langle n\rangle}{\alpha}\right)-\exp \left(-\frac{n /\langle n\rangle}{\beta}\right)\right]
$$

Here, $n$ is the number of monomers between two adjacent network nodes, and $\langle n\rangle$ is the average. $\alpha$ and $\beta$ are numerical parameters empirically determined by fitting. Uneyama ${ }^{60)}$ has derived the distribution function from the free energy of the slip-link network with assuming a repulsive interaction between network nodes. The approximated formula in the long chain limit is reported as follows.

$$
P(n)=\frac{25}{6}\left(\frac{10 n^{3}}{\pi\langle n\rangle^{5}}\right)^{1 / 2} \exp \left(-\frac{5 n}{2\langle n\rangle}\right)
$$

Greco ${ }^{61)}$ proposed a different functional form from the thermodynamics, yet the agreement with the data is compatible with eq. 6. As a consequence of $M_{\mathrm{e}}$ distribution, the network strand length and the number of entanglements on each chain also have distributions. Nevertheless, the effect of distributions on rheology and polymer dynamics has not been clarified yet. For instance, melt rheology can be reasonably reproduced by the molecular theories, both with and without the distribution of entanglement network.

Another interesting direction is the analysis of topological network under deformations. Baig et al. ${ }^{62)}$ elaborated to observe the modified topological network under steady shear for the full-atomistic simulation of polyethylene. They reported that the number of network nodes decreases with an increase of shear rate. A similar reduction of network nodes has been observed for some coarse-grained model ${ }^{63-65)}$. These results are in harmony with some theories, in which the entanglement density is assumed to decrease under fast deformations. The representative one is the tube theory proposed by Doi-Edwards ${ }^{66}$, who derived the damping function under large step deformations from the loss of entanglement

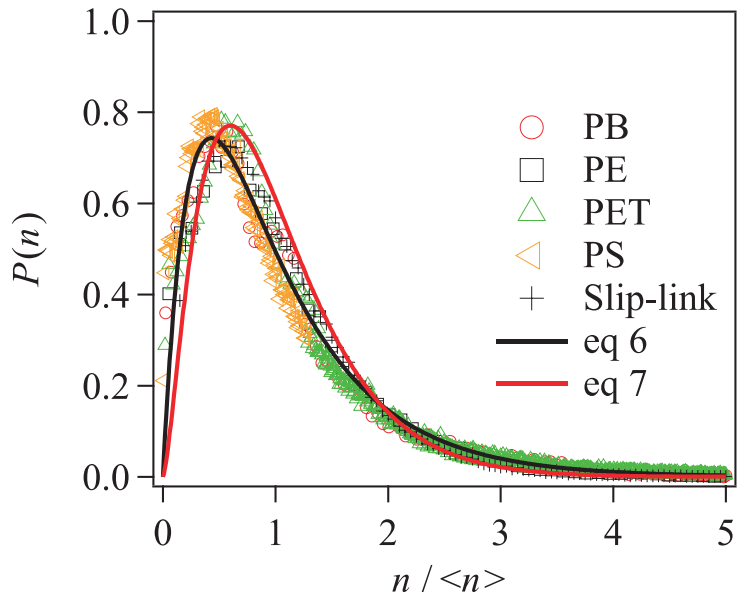

Fig. 3 Distribution of monomer number on the network strand. Unfilled symbols show the distribution for the topological network extracted from the snapshot of atomistic molecular simulations for various polymers ${ }^{53}$. Cross indicates the result from the multi-chain slip-link simulation $^{59)}$. Black and red curves show eqs. 6 and 7. For eq. 6 , $\alpha=0.77$ and $\beta=0.26$.

induced by the chain contraction. The so-called Q tensor expresses the non-linearity based on the loss of entanglement. Due to the remarkable success of the Doi-Edwards theory and the extended model ${ }^{67}$, the loss of entanglement is frequently discussed for non-linear rheology of entangled polymers. For instance, in the interrupted shear experiments ${ }^{68-73)}$, the reduced stress overshoot observed in the second start-up flow is analyzed in terms of the recovery of entanglement during the interval between consecutive flows. In the superposed shear experiments ${ }^{74-77)}$, in which oscillatory deformations are additionally applied to steady shear, a softening in the oscillatory response is related to the amount of disentanglement. Towards industrial direction, in some numerical simulations where the microscopic models are combined with macroscopic calculations, the spatial distribution of entanglement density has been reported in macroscopic flow channels $^{78,79)}$.

However, it must be noted that most of the rheological properties can be explained even if the entanglement density is unchanged. Marrucci et $a l .^{80)}$ have shown that the damping function derived from the force balance around entanglement (with a constant entanglement density) is very close to the Doi-Edwards prediction. The constitutive equation based on the theory by Marrucci et al ${ }^{81}$ reasonably reproduces a variety of non-linear viscoelastic phenomena. The other model developed by Graham et al ${ }^{82,83)}$ is also capable of semi-quantitative prediction with a constant entanglement density. These results imply that the modification of the entanglement network might be an artefact, and there would be other different explanations for the experimental data. 


\section{CONCLUDING REMARKS}

In this short review, we note that entanglement molecular weight is a parameter for a specific theoretical model, and not a material property intrinsically defined. Misleading analysis and discussion might be conducted if the value of $M_{\mathrm{e}}$ is given without the model description and the method of determination. For example, the $M_{\mathrm{e}}$ values for $A=1$ and $A=4 / 5$ must be discriminated. The characteristic time of the coarsegrained models, such as the entanglement time $\tau_{\mathrm{e}}$ in the tube model, must be consistently defined with $M_{\mathrm{e}}$, as mentioned by Larson et al. ${ }^{20)}$ Besides, the evaluation method of $G_{\mathrm{N}}$ should be indicated. Concerning molecular simulations, the topological analysis is widely used, and empirical consistency for the obtained value of $M_{\mathrm{e}}$ has been accumulated with the value from the other methods. However, we note that the relation between the obtained topology from frozen snapshots and the retarded polymer dynamics has not been revealed yet. Flow-induced modification of the topological network attracts attention, and the idea may stimulate developments in the polymer industry. Nevertheless, note that the related experimental observations for rheological response could be reproduced with the models that do not take account of the change of entanglement density.

\section{ACKNOWLEDGEMENT}

The authors thank the financial support from Ogasawara Foundation and JST-CREST (JPMJCR1992).

\section{REFERENCES}

1) Ferry JD, “Viscoelastic Properties of Polymers”, 3rd ed. (1980), John Wiley \& Sons, Inc.

2) Rouse PE, J Chem Phys, 21, 1272 (1953).

3) Doi M, Edwards SF, "The Theory of Polymer Dynamics", (1986), Clarendon press, Oxford.

4) Watanabe H, Prog Polym Sci, 24, 1253 (1999).

5) Graessley W, "Polymeric Liquids \& Networks: Dynamics and Rheology", (2008), Garland Science, London.

6) Baumgartner A, Binder K, J Chem Phys, 75, 6 (1981).

7) Kremer K, Grest GS, Carmesin I, Phys Rev Lett, 61, 566 (1988)

8) Iwata K, Edwards SF, J Chem Phys, 90, 4567 (1989).

9) Kavassalis TA, Noolandi J, Phys Rev Lett, 59, 2674 (1987).

10) Fetters LJ, Lohse DJ, Milner ST, Graessley WW, Macromolecules, 32, 6847 (1999).

11) Wu S, J Polym Sci Part B Polym Phys, 27, 723 (1989).

12) Lin YH, Macromolecules, 20, 3080 (1987).

13) Boek ES, Coveney PV, Lekkerkerker HNW, van der Schoot P,
Phys Rev E, 55, 3124 (1997).

14) Masubuchi Y, Annu Rev Chem Biomol Eng, 5, 11 (2014).

15) Masubuchi Y, In:Reference Module in Materials Science and Materials Engineering, Elsevier, 2016: 1.

16) Green MS, Tobolsky AV, J Chem Phys, 14, 80 (1946).

17) Masubuchi Y, Takimoto J-I, Koyama K, Ianniruberto G, Marrucci G, Greco F, J Chem Phys, 115, 4387 (2001).

18) de Gennes PG, J Chem Phys, 55, 572 (1971).

19) Doi M, Edwards SF, J Chem Soc Faraday Trans 2, 74, 1789 (1978).

20) Larson RG, Sridhar T, Leal LG, McKinley GH, Likhtman AE, McLeish TCB, J Rheol, 47, 809 (2003).

21) Everaers R, New J Phys, 1, 12.1 (1999).

22) Rubinstein M, Panyukov S, Macromolecules, 35, 6670 (2002).

23) Flory PJ, "Principles of Polymer Chemistry", (1953), Cornell University Press, Ithaca, New York.

24) James HM, Guth E, J Chem Phys, 15, 669 (1947).

25) Flory PJ, Proc $R$ Soc London A Math Phys Sci, 351, 351 (1976).

26) Fetters LJ, Lohse DJ, Richter D, Witten TA, Zirkel A, Macromolecules, 27, 4639 (1994).

27) Masubuchi Y, Ianniruberto G, Greco F, Marrucci G, J Chem Phys, 119, 6925 (2003).

28) Masubuchi Y, Ianniruberto G, Greco F, Marrucci G, J NonNewton Fluid Mech, 149, 87 (2008).

29) Uneyama T, Masubuchi Y, J Chem Phys, 137, 154902 (2012).

30) Chappa VC, Morse DC, Zippelius A, Müller M, Phys Rev Lett, 109, 148302 (2012).

31) Ramírez-Hernández A, Detcheverry FA, Peters BL, Chappa VC, Schweizer KS, Müller M, de Pablo JJ, Macromolecules, 46, 6287-6299 (2013).

32) Langeloth M, Masubuchi Y, Böhm MC, Müller-Plathe F, $J$ Chem Phys, 138, 104907 (2013).

33) Uneyama T, Masubuchi Y, in preparation.

34) Masubuchi Y, Uneyama T, Soft Matter, 14, 5986 (2018).

35) Masubuchi Y, Macromolecules, 51, 10184 (2018).

36) Likhtman AE, McLeish TCB, Macromolecules, 35, 63326343 (2002).

37) Auhl D, Ramirez J, Likhtman AE, Chambon P, Fernyhough C, J Rheol, 52, 801 (2008).

38) Likhtman AE, Sukumaran SK, Ramirez J, Macromolecules, 40, 6748 (2007).

39) Masubuchi Y, Uneyama T, Korea Aust Rheol J, 31, 241 (2019).

40) Kremer K, Grest GS, J Chem Phys, 92, 5057 (1990).

41) Liu C, He J, van Ruymbeke E, Keunings R, Bailly C, Polymer, 47, 4461 (2006).

42) Marvin RS, Oser H, J Res Natl Bur Stand Sect B Math Math Phys, 66B, 171 (1962).

43) Flory PJ, Chem Rev, 35, 51 (1944). 
44) Doi M, J Polym Sci Polym Phys Ed, 21, 667 (1983).

45) Fuchs K, Friedrich C, Weese J, Macromolecules, 29, 5893 (1996).

46) Liu C, Halasa AF, Keunings R, Bailly C, Macromolecules, 39, 7415 (2006).

47) van Ruymbeke E, Vlassopoulos D, Kapnistos M, Liu CY, Bailly C, Macromolecules, 43, 525 (2010).

48) Masubuchi Y, Nihon Reoroji Gakkaishi (J Soc Rheol Jpn), 48, 37 (2020).

49) Pütz M, Kremer K, Grest GS, Europhys Lett, 49, 735 (2000).

50) Everaers R, Sukumaran SK, Grest GS, Svaneborg C, Sivasubramanian A, Kremer K, Science, 303, 823 (2004).

51) Sukumaran SK, Grest GS, Kremer K, Everaers R, J Polym Sci Part B Polym Phys, 43, 917 (2005).

52) Kroger M, Comput Phys Commun, 168, 209 (2005).

53) Tzoumanekas C, Theodorou DN, Macromolecules, 39, 4592 (2006).

54) Shanbhag S, Larson RG, Macromolecules, 39, 2413 (2006).

55) Everaers R, Phys Rev E - Stat Nonlinear, Soft Matter Phys, 86, 1 (2012).

56) Bisbee W, Qin J, Milner ST, Macromolecules, 44, 8972 (2011).

57) de Gennes PG, J Phys, 36, 1199 (1975).

58) Schieber JD, J Chem Phys, 118, 5162 (2003).

59) Masubuchi $Y$, Uneyama $T$, Watanabe $H$, Ianniruberto $G$, Greco F, Marrucci G, J Chem Phys, 132, 134902 (2010).

60) Uneyama T, Masubuchi Y, J Chem Phys, 135, 184904 (2011).

61) Greco F, Eur Phys J E, 25, 175 (2008).

62) Baig C, Mavrantzas VG, Kröger M, Macromolecules, 43, 6886 (2010).

63) Masubuchi Y, Watanabe H, Ianniruberto G, Greco F, Marrucci G, Nihon Reoroji Gakkaishi (J Soc Rheol Jpn), 32, 197 (2004).

64) Masubuchi Y, J Chem Phys, 143, 224905 (2015).

65) Sgouros AP, Megariotis G, Theodorou DN, Macromolecules,

\section{0, 4524 (2017).}

66) Doi M, Edwards SF, J Chem Soc Faraday Trans 2, 74, 1802 (1978).

67) Mead DW, Larson RG, Doi M, Macromolecules, 31, 7895 (1998).

68) Dealy JM, Tsang W, J Appl Polym Sci, 26, 1149 (1981).

69) Menezes E, Graessley W, J Polym Sci Polym Phys Ed, 20, 1817 (1982).

70) Sanchez-Reyes J, Archer LA, J Rheol, 47, 469 (2003).

71) Robertson CG, Warren S, Plazek DJ, Roland CM, Macromolecules, 37, 10018 (2004).

72) Wang SQ, Wang $Y$, Cheng S, Li X, Zhu X, Sun H, Macromolecules, 46, 3147 (2013).

73) Roy D, Roland CM, Macromolecules, 46, 9403 (2013).

74) Isayev AI, Wong CM, J Polym Sci Part B Polym Phys, 26, 2303 (1988).

75) Wong CM, Isayev AI, Rheol Acta, 28, 176 (1989).

76) Somma E, Valentino O, Titomanlio G, Ianniruberto G, $J$ Rheol, 51, 987 (2007).

77) Vermant J, Walker L, Moldenaers P, Mewis J, J Non-Newton Fluid Mech, 79, 173 (1998).

78) Murashima T, Taniguchi T, Europhysics Lett, 96, 18002 (2011).

79) Sato T, Harada K, Taniguchi T, Macromolecules, 52, 547 (2019).

80) Marrucci G, Greco F, Ianniruberto G, Macromol Symp, 158, 57 (2000).

81) Marrucci G, Greco F, Ianniruberto G, Rheol Acta, 40, 98 (2001).

82) Graham RS, Likhtman AE, McLeish TCB, Milner ST, $J$ Rheol, 47, 1171 (2003).

83) Likhtman AE, Graham RS, J Non-Newton Fluid Mech, 114, 1 (2003). 